\title{
Linear mappings preserving similarity on $B(H)$
}

by

TAtJana Petek (Maribor)

\begin{abstract}
Let $H$ be an infinite-dimensional complex Hilbert space. We give a characterization of surjective linear mappings on $B(H)$ that preserve similarity in both directions.
\end{abstract}

The problem we consider is one of the so-called linear preserver problems that have attracted a lot of attention in recent decades. In these problems one is interested in characterizing linear mappings on some algebra of operators that leave certain functions, subsets, relations, etc., invariant. A lot of results of this kind can be found in the survey papers [9], [1], [2] and the references therein.

Let $M_{n}(\mathbb{F})$ denote the algebra of all $n \times n$ matrices with entries in $\mathbb{F}$. Linear similarity-preserving mappings on $M_{n}(\mathbb{F})$ (i.e. such that the similarity of $A, B \in M_{n}(\mathbb{F})$ implies the similarity of $\phi(A)$ and $\left.\phi(B)\right)$ were characterized by Hiai [3] and Lim [10].

THEOREM 1. Let $\phi$ be a linear mapping on $M_{n}(\mathbb{F})$, where $\mathbb{F}$ is an infinite field such that char $\mathbb{F}=0$ or char $\mathbb{F}$ does not divide $n$. Then $\phi$ is similaritypreserving if and only if one of the following holds:

(a) there exists $B \in M_{n}(\mathbb{F})$ such that

$$
\phi(X)=\operatorname{tr}(X) B, \quad X \in M_{n}(\mathbb{F}) ;
$$

(b) there exist a nonsingular matrix $A \in M_{n}(\mathbb{F})$ and $c, d \in \mathbb{F}$ such that either

$$
\phi(X)=c A X A^{-1}+d \operatorname{tr}(X) I, \quad X \in M_{n}(\mathbb{F}),
$$

or

$$
\phi(X)=c A X^{t} A^{-1}+d \operatorname{tr}(X) I, \quad X \in M_{n}(\mathbb{F}) .
$$

Here $\operatorname{tr}(X)$ denotes the trace and $X^{t}$ the transpose. Similar problems of preserving other equivalence relations (unitary equivalence, consimilarity etc.) were considered in [4].

Key words and phrases: Hilbert space, similarity-preserving mapping. 
It turns out that similarity preservers are connected with nilpotent preservers. If a linear map $\phi: M_{n}(\mathbb{F}) \rightarrow M_{n}(\mathbb{F})$ preserves similarity, then it preserves nilpotents [10]. Indeed, if $N$ is nilpotent, then $N \sim 2 N$, which implies $\phi(N) \sim 2 \phi(N)$. Then the only eigenvalue of $\phi(N)$ is zero, and therefore $\phi(N)$ is nilpotent.

We will study similarity-preserving mappings on $B(H)$, the algebra of all bounded linear operators on the infinite-dimensional complex Hilbert space $H$. The operators $A$ and $B$ are similar $(A \sim B)$ if there exists an invertible operator $S \in B(H)$ such that $A=S B S^{-1}$. We say that a linear mapping $\phi: B(H) \rightarrow B(H)$ preserves similarity if the similarity of any $A$ and $B$ implies the similarity of $\phi(A)$ and $\phi(B)$, and it preserves similarity in both directions whenever $A \sim B$ if and only if $\phi(A) \sim \phi(B)$. In the case that $\phi$ is bijective, $\phi$ preserves similarity in both directions if $\phi$ and $\phi^{-1}$ preserve similarity. The set of operators that are similar to a given $A$ will be called the similarity orbit and denoted by $\mathcal{S}(A)$.

In this note, operators of rank one, especially nilpotents of rank one will be of importance. By $x \otimes y$ we denote the bounded linear operator acting as $(x \otimes y) z=\langle z, y\rangle x, x \in H$. Clearly, $x \otimes y$ is a nilpotent of rank one if and only if $x$ and $y$ are non-zero and $\langle x, y\rangle=0$. It is also easy to see that all rank one nilpotents are similar to each other.

Let us now state our main theorem.

Theorem 2. Let $H$ be an infinite-dimensional complex Hilbert space. If a surjective linear map $\phi: B(H) \rightarrow B(H)$ preserves similarity in both directions then there exist a non-zero complex number $c$ and a bounded bijective linear operator $A: H \rightarrow H$ such that either

(i) $\phi(T)=c A T A^{-1}$ for every $T \in B(H)$, or

(ii) $\phi(T)=c A T^{t} A^{-1}$ for every $T \in B(H)$,

where $T^{t}$ denotes the transpose of $T$ relative to a fixed but arbitrary orthonormal basis.

Many of the linear preserver problems can be translated to the problem of preserving rank one operators. For example, spectrum-preserving linear or additive maps on $B(X)$, the algebra of all bounded linear operators on a complex Banach space $X$, have been treated in [5] and [12] where the proof is based on spectrum characterizations of rank one operators. Similarly, in [14] a characterization of rank one nilpotents in terms of nilpotents was obtained.

We prove our theorem by characterizing rank one nilpotents in $B(H)$ in terms of similarity. From now on we assume that $\phi$ is as in Theorem 2. Let us first show that a surjective mapping $\phi$ that preserves similarity in both directions is bijective. 
LEMMA 3. If a linear map $\phi: B(H) \rightarrow B(H)$ preserves similarity in both directions, then it is one-to-one.

Proof. Suppose $\phi(X)=0$. As $A \sim 0$ if and only if $A=0$, we get $\phi(X) \sim 0=\phi(0)$, which implies that $X \sim 0$, and hence $X=0$.

In the finite-dimensional case, nilpotent matrices $N$ are exactly those that are similar to $2 N$. For $N \in B(H)$ we will show that similarity of $N$ and $2 N$ implies nilpotency of $N$. While the converse is also true if rank $N$ is finite we do not know whether it holds for nilpotents of infinite rank.

LEMMA 4. Every $N \in B(H)$ which is similar to $2 N$ is nilpotent.

Proof. Suppose $2 N=S^{-1} N S$ for some invertible $S \in B(H)$. Then $2^{k} N^{k}=S^{-1} N^{k} S$ for every positive integer $k$. Taking the norms we arrive at

$$
\left(2^{k}-\left\|S^{-1}\right\|\|S\|\right)\left\|N^{k}\right\| \leq 0 \quad \text { for every } k,
$$

which implies that $N$ must be nilpotent.

In the next lemma we characterize rank one nilpotents in terms of similarity.

LEMMA 5. For any non-zero operator $N \in B(H)$ the following assertions are equivalent.

(i) $N$ is a rank one nilpotent.

(ii) $N \sim 2 N$, and for every $A \in \mathcal{S}(N)$ which is not a multiple of $N$, if $A+N \sim N$ then $A+\lambda N \sim N$ for every $\lambda \in \mathbb{C}$.

Proof. Let $N=x \otimes y$ be a rank one nilpotent. Clearly, $N \sim 2 N$. Let now $A \notin \operatorname{span}\{N\}$ be similar to $N$. This implies that $A$ is also a rank one nilpotent and can thus be written as $u \otimes v$ for some non-zero $u, v \in H$ satisfying $\langle u, v\rangle=0$. Suppose $A+N \sim N$. From the fact that $A+N=$ $u \otimes v+x \otimes y$ is a rank one operator it follows that either $x$ and $u$ are linearly dependent, or $v$ and $y$ are linearly dependent. Let $u=k x$ for some $k \neq 0$. Then $A+N=x \otimes(k v+y)$ and for every $\lambda \in \mathbb{C}$ we also have $A+\lambda N=x \otimes(k v+\lambda y)$, which is a non-zero rank one operator since $A$ is not proportional to $N$. Since $\langle x, k v+\lambda y\rangle=0$ it follows that $A+\lambda N$ is a rank one nilpotent and thus similar to $N$. The case that $v$ and $y$ are linearly dependent is analogous. We have shown that a rank one nilpotent $N$ satisfies (ii).

Now, we prove that (ii) implies (i). If $N$ is not a rank one nilpotent and $N \sim 2 N$ then it is a nilpotent of rank greater than 1 . We will exhibit an $A \in \mathcal{S}(N), A \notin \operatorname{span}\{N\}$, such that $A+N \sim N$, but $A-N \notin \mathcal{S}(N)$. 
First of all assume $N^{2}=0$ and decompose $H=\operatorname{Ker} N \oplus(\operatorname{Ker} N)^{\perp}$. Choose a non-zero $y \in(\operatorname{Ker} N)^{\perp}=Y$, let $Y_{0}=\operatorname{span}\{y\}$ and split $H=$ Ker $N \oplus\left(Y \ominus Y_{0}\right) \oplus Y_{0}$. The operator matrix form of $N$ then is

$$
N=\left[\begin{array}{ccc}
0 & M_{1} & m_{2} \\
0 & 0 & 0 \\
0 & 0 & 0
\end{array}\right], \quad M_{1} \neq 0, m_{2} \neq 0
$$

Note that $m_{2}$ is a rank one operator. Let

$$
A=\left[\begin{array}{ccc}
0 & M_{1} & 2 m_{2} \\
0 & 0 & 0 \\
0 & 0 & 0
\end{array}\right]
$$

Clearly, $A$ is bounded and similar to $N$, for

$$
A=S^{-1} N S, \quad S=\left[\begin{array}{ccc}
I & 0 & 0 \\
0 & I & 0 \\
0 & 0 & 2
\end{array}\right]
$$

Also, the sum

$$
A+N=\left[\begin{array}{ccc}
0 & 2 M_{1} & 3 m_{2} \\
0 & 0 & 0 \\
0 & 0 & 0
\end{array}\right]=\left(S^{\prime}\right)^{-1} N S^{\prime}, \quad S^{\prime}=\left[\begin{array}{ccc}
I & 0 & 0 \\
0 & 2 I & 0 \\
0 & 0 & 3
\end{array}\right]
$$

is similar to $N$. However,

$$
A-N=\left[\begin{array}{ccc}
0 & 0 & m_{2} \\
0 & 0 & 0 \\
0 & 0 & 0
\end{array}\right]
$$

is not similar to $N$ as $\operatorname{rank}(A-N)$ is one.

Assume now that $N$ is a nilpotent of nilindex $k>2$. Then there exists a $u \neq 0$ such that $u, N u, \ldots, N^{k-1} u$ are linearly independent. Let $Y=$ $\operatorname{span}\left\{u, N u, \ldots, N^{k-1} u\right\}$. According to the decomposition $H=Y \oplus Y^{\perp}$ we can represent $N$ as

$$
N=\left[\begin{array}{ll}
J & N_{2} \\
0 & N_{3}
\end{array}\right]
$$

where $J$ is the transpose of a $k \times k$ Jordan block.

Define $S_{X}=\left[\begin{array}{cc}I & X \\ 0 & I\end{array}\right]$ for any bounded linear operator $X: Y^{\perp} \rightarrow Y$. Evidently, $S_{X}$ is invertible and $S_{X}^{-1}=S_{-X}$. Let us compute 


$$
\begin{gathered}
S_{X}^{-1} N S_{X}=N+\left[\begin{array}{cc}
0 & J X-X N_{3} \\
0 & 0
\end{array}\right], \\
S_{2 X}^{-1} N S_{2 X}=N+\left[\begin{array}{cc}
0 & 2 J X-2 X N_{3} \\
0 & 0
\end{array}\right], \\
S_{X}^{-1} 2 N S_{X}=2 N+\left[\begin{array}{cc}
0 & 2 J X-2 X N_{3} \\
0 & 0
\end{array}\right] .
\end{gathered}
$$

Let us show that there is a rank one operator $X$ such that $J X-X N_{3} \neq 0$. If $N_{3}=0$ then take any unit vector $w \in Y^{\perp}$ and define $X y=\langle y, w\rangle u$ for $y \in Y^{\perp}$. Then $\left(J X-X N_{3}\right) w=J u=N u \neq 0$. If $N_{3} \neq 0$ there exists a unit vector $w \in \operatorname{Im} N_{3}$ such that $w=N_{3} x$ for some $x \in Y^{\perp}$. Define $X y=\langle y, w\rangle N^{k-1} u$ for $y \in Y^{\perp}$. Since $N^{k} u=0$ we obtain

$$
\begin{aligned}
\left(J X-X N_{3}\right) x & =J\langle x, w\rangle N^{k-1} u-\left\langle N_{3} x, w\right\rangle N^{k-1} u \\
& =\langle x, w\rangle N^{k} u-N^{k-1} u=-N^{k-1} u \neq 0 .
\end{aligned}
$$

Finally, by choosing

$$
A=N+\left[\begin{array}{cc}
0 & 2 J X-2 X N_{3} \\
0 & 0
\end{array}\right]=S_{2 X}^{-1} N S_{2 X}
$$

we achieve that

$$
A+N=S_{X}^{-1} 2 N S_{X} \sim 2 N \sim N
$$

but $A-N$ cannot be similar to $N$ as $(A-N)^{2}=0$ while $N^{2} \neq 0$.

Let us see how we can make use of this characterization. We will show that $\phi$ preserves rank one nilpotents in both directions. Let $N$ be a nilpotent of rank one. Then $N \sim 2 N$, which implies that $\phi(N)$ is similar to $2 \phi(N)$, and consequently, $\phi(N)$ is nilpotent. Let $A \in B(H), A \notin \operatorname{span}\{\phi(N)\}, A \sim \phi(N)$ and $A+\phi(N) \sim \phi(N)$. Since $\phi$ is bijective there exists a $B \in B(H)$ such that $\phi(B)=A$ and $B$ is not a multiple of $N$. As $\phi$ preserves similarity in both directions, $B \sim 2 B$ and therefore $B$ is nilpotent. The relations $A \sim \phi(N)$, $A+\phi(N) \sim \phi(N)$, linearity of $\phi$ and the fact that $\phi$ preserves similarity in both directions imply that $B \sim N$ and $B+N \sim N$. By Lemma 5(ii), $B+\lambda N \sim N$ for every $\lambda \in \mathbb{C}$. Thus $\phi(B+\lambda N)=A+\lambda \phi(N) \sim \phi(N)$ for every $\lambda \in \mathbb{C}$, which shows that $\phi(N)$ is a rank one nilpotent. As $\phi$ is bijective and preserves similarity in both directions, it preserves rank one nilpotents in both directions.

Before we proceed we need some more technical lemmas.

Lemma 6. Let $K$ be a complex Hilbert space (finite- or infinite-dimensional) and $B \in B(K)$. The following are equivalent: 
(1) There is no finite-rank nilpotent $N \in B(K)$ with nilindex 2 such that $B+N$ is similar to $B$.

(2) $B=\beta I$ for some $\beta \in \mathbb{C}$.

Proof. Clearly (2) implies (1). Suppose now that $B$ is not scalar. Then obviously, $\operatorname{dim} K>1$. If $K$ is of finite dimension then up to similarity we can represent $B$ in its Jordan form as

$$
B=\left[\begin{array}{cc}
J_{1} & 0 \\
0 & J_{2}
\end{array}\right]
$$

where $J_{1}=\left[\begin{array}{ll}a & 0 \\ 0 & b\end{array}\right], a \neq b$, or $J_{1}$ is a $k \times k$ Jordan block, $2 \leq k \leq \operatorname{dim} K$, and the matrix $J_{2}$ may be missing. In both cases, taking as $N$ the matrix having 1 in (1,2)-position and zeros elsewhere gives the similarity of $B+N$ and $B$. Note that $N$ is a rank one square-zero matrix.

Let now $K$ be infinite-dimensional. As $B$ is not scalar there exists $x \in K$ such that $x$ and $B x$ are linearly independent. Denote by $U$ the orthogonal complement of $\operatorname{span}\{x, B x\}$ in $K$. According to this decomposition $B$ has now the form

$$
\left[\begin{array}{ll}
B_{11} & B_{12} \\
B_{21} & B_{22}
\end{array}\right], \quad B_{11}=\left[\begin{array}{ll}
0 & * \\
1 & *
\end{array}\right], \quad B_{21} x=0 .
$$

Let us choose a unit vector $y \in\left(\operatorname{Im} B_{21}\right)^{\perp}$ and define the operators

$$
X: U \rightarrow \operatorname{span}\{x, B x\}, \quad X u=\langle u, y\rangle x, \quad S=\left[\begin{array}{cc}
I & X \\
0 & I
\end{array}\right] \text {. }
$$

Then

$$
S^{-1} B S=B+\left[\begin{array}{cc}
-X B_{21} & B_{11} X-X B_{21} X-X B_{22} \\
0 & B_{21} X
\end{array}\right] .
$$

Simple calculation shows that $X B_{21}=0$ and $B_{21} X=0$, so

$$
S^{-1} B S=B+N, \quad N=\left[\begin{array}{cc}
0 & B_{11} X-X B_{22} \\
0 & 0
\end{array}\right] .
$$

Applying linear independence of $x$ and $B x$ we observe that $N$ is non-zero,

$$
\left(B_{11} X-X B_{22}\right) y=\langle y, y\rangle B_{11} x-\left\langle B_{22} y, y\right\rangle x=B x-\left\langle B_{22} y, y\right\rangle x,
$$

and since $N$ is a sum of two operators of rank at most one, its rank cannot exceed 2. Clearly, $N^{2}=0$. So, we have found a non-zero square-zero nilpotent $N$ of rank at most 2 such that $B+N$ is similar to $B$.

Lemma 7. Let $P \in B(H)$ be a non-trivial $(\neq 0, I)$ idempotent and $B$ a bounded linear operator on $H$. If for every finite rank nilpotent $N$,

$$
P+N \sim P \text { if and only if } B+N \sim B
$$


then there exist constants $\alpha, \beta, \alpha \neq 0$, such that

$$
B=\alpha P+\beta I .
$$

Proof. It is easy to see that every idempotent is similar to a projection. Therefore, there is no loss of generality in assuming that $P$ is a non-trivial projection. There exists a decomposition $H=U \oplus V$ into closed orthogonal non-trivial subspaces $U$ and $V$ such that

$$
P=\left[\begin{array}{ll}
I & 0 \\
0 & 0
\end{array}\right], \quad B=\left[\begin{array}{ll}
B_{11} & B_{12} \\
B_{21} & B_{22}
\end{array}\right] .
$$

We first show that if (2) holds then $B_{12}$ and $B_{21}$ must be zero. This is equivalent to the fact that $U$ and $V$ are invariant subspaces for $B$. Choose non-zero $e \in U$ and $f \in V$. Then $e \otimes f$ is a rank one nilpotent. Let $\beta \in \mathbb{C}$ and define

$$
X_{\beta}: V \rightarrow U, \quad X_{\beta} v=\beta(e \otimes f) v=\beta\langle v, f\rangle e, \quad v \in V,
$$

and

$$
S_{\beta}=\left[\begin{array}{cc}
I & X_{\beta} \\
0 & I
\end{array}\right] .
$$

It is easy to see that $P+\beta e \otimes f=S_{\beta}^{-1} P S_{\beta}$ and so, by (2), $B+\beta e \otimes f$ is similar to $B$ for every $\beta$. We will now use Lemma 4 from [5] which states that if $\alpha \notin \sigma(B)$ then

$$
\alpha \text { is an eigenvalue of } B+e \otimes f \Leftrightarrow\left\langle(\alpha I-B)^{-1} e, f\right\rangle=1 .
$$

Choose $\alpha>\|B\|$ and suppose that $\left\langle(\alpha I-B)^{-1} e, f\right\rangle \neq 0$. There exists a non-zero constant $\beta$ such that $\left\langle(\alpha I-B)^{-1} \beta e, f\right\rangle=1$. This shows that $\alpha$ is an eigenvalue of $B+\beta e \otimes f$ and leads to a contradiction with $B+\beta e \otimes f \sim B$. So, for every $e \in U$ and $f \in V$ we have $\left\langle(\alpha I-B)^{-1} e, f\right\rangle=0$. It follows that $(\alpha I-B)^{-1} U \subseteq V^{\perp}=U$, so $U$ is invariant for $(\alpha I-B)^{-1}$. Taking $e \in V$ and $f \in U$ and applying similar arguments we find that $V$ is also invariant for $(\alpha I-B)^{-1}$ for all $\alpha>\|B\|$. Therefore, $U$ and $V$ are both invariant for $\alpha I-B$, and finally for $B$. The operator $B$ has now the form

$$
B=\left[\begin{array}{cc}
B_{11} & 0 \\
0 & B_{22}
\end{array}\right] \text {. }
$$

It remains to show that $B_{11}$ and $B_{22}$ are multiples of identity. We will prove this only for $B_{11} \in B(U)$; the argument for $B_{22}$ is similar. Assuming that $B_{11}$ is not scalar and applying Lemma 6 we can find a rank one or two nilpotent $N \in B(U), N^{2}=0$, such that $B_{11}+N \sim B_{11}$, and consequently $B+(N \oplus 0) \sim B$; but $P+(N \oplus 0)$ is not idempotent, and therefore it cannot be similar to $P$. The fact that $\alpha \neq 0$ follows from non-triviality of $P$. 
We already know that $\phi$ preserves rank one nilpotents in both directions. By standard methods (see [14, pp. 531-533]) one can find a bounded invertible linear operator $A$ and a non-zero constant $c$ such that either

or

$$
\phi(x \otimes y)=c A(x \otimes y) A^{-1} \quad \text { for all } x, y \in H,\langle x, y\rangle=0,
$$

$$
\phi(x \otimes y)=c A(y \otimes x) A^{-1} \quad \text { for all } x, y \in H,\langle x, y\rangle=0 .
$$

By linearity of $\phi$ and by composing $\phi$ with transposition if necessary, we may now assume with no loss of generality that

$$
\phi(N)=N
$$

for all finite rank nilpotents $N \in B(H)$.

Let us show that for every non-trivial idempotent $P$ there exist constants $\alpha$ and $\beta$ such that $\phi(P)=\alpha P+\beta I$. Let $N$ be any finite rank nilpotent. Then

$$
\begin{aligned}
P+N \sim P & \Leftrightarrow \phi(P)+\phi(N) \sim \phi(P) \\
& \Leftrightarrow \phi(P)+N \sim \phi(P),
\end{aligned}
$$

and Lemma 7 gives the desired conclusion.

Next, we show that $\alpha=1$ for every finite rank idempotent $P$. As $P$ is not scalar there exists a finite rank nilpotent $N$ such that $P+N \sim P$. Therefore

$$
\phi(P)=\alpha P+\beta I, \quad \phi(P+N)=\alpha^{\prime}(P+N)+\beta^{\prime} I,
$$

and, on the other hand,

$$
\begin{aligned}
\phi(N) & =N=\phi(P+N)-\phi(P) \\
& =\alpha^{\prime} N+\left(\alpha^{\prime}-\alpha\right) P+\left(\beta^{\prime}-\beta\right) I
\end{aligned}
$$

and $\left(1-\alpha^{\prime}\right) N=\left(\alpha^{\prime}-\alpha\right) P+\left(\beta^{\prime}-\beta\right) I$, which implies $\alpha=\alpha^{\prime}=1, \beta^{\prime}=\beta$. We have thus arrived at

$$
\phi(P)=P+k \beta I
$$

for every rank $k$ idempotent $P$.

We also have $\phi(P)=P+\mu_{P} I$ for any idempotent $P \neq I$ of infinite rank. To verify this, choose any non-zero finite-rank idempotent $Q$ satisfying $P Q=Q P=Q$. Then $P-Q$ is a non-trivial idempotent. So, there exist constants $\alpha_{1}, \alpha_{2}, \mu_{1}, \mu_{2}, \mu_{3}$ such that

$$
\begin{aligned}
\phi(Q) & =Q+\mu_{1} I, \\
\phi(P) & =\alpha_{1} P+\mu_{2} I, \\
\phi(P-Q) & =\alpha_{2}(P-Q)+\mu_{3} I .
\end{aligned}
$$

Applying linearity of $\phi$ gives $\alpha_{1}=\alpha_{2}=1$, and so

$$
\phi(P)=P+\mu_{P} I
$$

for every non-trivial idempotent $P$. 
Finally, we use the result of Pearcy and Topping [13] which states that every operator on $B(H)$ is a sum of five idempotents, and infer that $\phi(X)=$ $X+f(X) I$ for every $X \in B(H)$ and some linear functional $f$ on $B(H)$. Every operator on $B(H)$ is also a finite sum of square-zero operators [13]. Verifying that $f(N)=0$ if $N^{2}=0$ then yields $\phi(X)=X$ for every $X \in B(H)$. Let us now show that every square-zero operator $N$ is similar to $2 N$. If we decompose $H=\operatorname{Ker} N \oplus(\operatorname{Ker} N)^{\perp}$, then the nilpotent $N$ takes the form

$$
N=\left[\begin{array}{cc}
0 & N_{1} \\
0 & 0
\end{array}\right]
$$

as $\operatorname{Im} N \subseteq \operatorname{Ker} N$. Taking $S=I \oplus 2 I$ we obtain $S^{-1} N S=2 N$, so $N \sim 2 N$. Thus $\phi(N) \sim 2 \phi(N), \phi(N)$ is nilpotent, and therefore $f(N)=0$. This completes the proof of Theorem 2 .

Let us finally note that Theorem 2 does not hold with the Hilbert space $H$ replaced by a Banach space $X$. Recall that there exists an infinitedimensional Banach space $X$ such that the algebra $B(X)$ has a non-zero multiplicative linear functional $f[11,15]$. Obviously, the mapping $\phi: B(X) \rightarrow$ $B(X)$ defined by $\phi(X)=X+f(X) I$ preserves similarity in both directions.

Recently, Ji and Du [8] obtained a similar characterization of mappings preserving similarity in both directions, but their assumptions were stronger: they assumed that $H$ is also separable and that $\phi$ is bounded. Related material can also be found in $[6,7]$.

\section{References}

[1] M. Brešar and P. Šemrl, Linear preservers on $B(X)$, in: Banach Center Publ. 38, Inst. Math., Polish Acad. Sci., 1997, 49-58.

[2] A. Guterman, C.-K. Li and P. Šemrl, Some general techniques on linear preserver problems, Linear Algebra Appl. 315 (2000), 61-81.

[3] F. Hiai, Similarity preserving linear maps on matrices, ibid. 97 (1987), 127-139.

[4] R. Horn, C.-K. Li and N.-K. Tsing, Linear operators preserving certain equivalence relations on matrices, SIAM J. Matrix Anal. Appl. 12 (1991), 195-204.

[5] A. A. Jafarian and A. R. Sourour, Spectrum-preserving linear maps, J. Funct. Anal. 66 (1986), 225-261.

[6] G. X. Ji, Similarity-preserving linear maps on $\mathcal{B}(\mathcal{H})$, Linear Algebra Appl. 360 (2003), 249-257.

[7] -, Asymptotic similarity-preserving linear maps on $\mathcal{B}(\mathcal{H})$, ibid. 368 (2003), 371-378.

[8] G. X. Ji and H. K. Du, Similarity-invariant subspaces and similarity-preserving linear maps, Acta Math. Sinica 18 (2002), 489-498.

[9] C.-K. Li and N.-K. Tsing, Linear preserver problems: A brief introduction and some special techniques, Linear Algebra Appl. 162-164 (1992), 217-235.

[10] M. H. Lim, A note on similarity preserving linear maps on matrices, ibid. 190 (1993) 229-233. 
[11] B. S. Mityagin and I. S. Edel'shteĭn, Homotopy type of linear groups for two classes of Banach algebras, Funktsional. Anal. i Prilozhen. 4 (1970), no. 3, 61-72 (in Russian).

[12] M. Omladič and P. Šemrl, Spectrum-preserving additive maps, Linear Algebra Appl. 153 (1991), 67-72.

[13] C. Pearcy and D. Topping, Sums of small numbers of idempotents, Michigan Math. J. 14 (1967), 453-465.

[14] P. Šemrl, Linear maps that preserve the nilpotent operators, Acta Sci. Math. (Szeged) 61 (1995), 523-534.

[15] A. Wilansky, Subalgebras of B(X), Proc. Amer. Math. Soc. 29 (1971), 355-360.

Faculty of Electrical Engineering and Computer Science

University of Maribor

2000 Maribor, Slovenia

E-mail: tatjana.petek@uni-mb.si

Received December 23, 2002

Revised version July 16, 2003 